\title{
Performance Optimization of Multicast Content Delivery in a Mobile Environment based on PMIPv6
}

\author{
Tien-Thinh Nguyen, Christian Bonnet \\ Department of Mobile Communications \\ EURECOM \\ Sophia-Antipolis, France \\ E-mail: \{Tien-Thinh.Nguyen, Christian.Bonnet\}@eurecom.fr
}

\begin{abstract}
Recently, a base solution has been adopted for supporting multicast listener mobility in Proxy Mobile IPv6 (PMIPv6). This solution brings multicast listener support into PMIPv6 by placing multicast routing functions at LMA while MAGs provide MLD proxy functions. Nevertheless, it does not address specific optimizations and performances issues such as handover latency, tunnel overhead, non-route optimization, etc. Specially, this paper focuses on handover performance in terms of service disruption time. The theoretical and simulation results show that through the utilization of multicast context transfer the service disruption time can be reduced significantly. By tuning the behavior of the IGMP/MLD for routers, we can also achieve a similar result, but make a dramatically increasing multicastsignaling. Thus, the impact of multicast-related signaling on the wireless link is studied to suggest the maximum number of listeners supported by one MAG. An enhanced multicast context transfer function is also proposed for group multicast mobility to reduce the number of signaling mess ages.
\end{abstract}

Keywords-IP multicast; multicast mobility; multicast context transfer; explicit tracking; tuning the behavior of MLD; PMIPv6 testbed; NS-3; handover delay.

\section{INTRODUCTION}

Currently, Internet video traffic continues to grow at a rapid pace especially in Mobile environment. Thus, how to efficiently distribute this type of traffic becomes one of the key questions. In this context, IP multicast, which provides an effective mechanism for video delivery, plays a very important role in the mobile environments.

Regarding the IP mobility, Proxy Mobile IPv6 (PMIPv6) [1] takes advantage of the network-based mobility management that provides mobility support for moving hosts without their involvement. Two possible approaches of multicast mobility support in PMIPv6 are considered and can be derived for both the source and the listener: (i) tunnel-based: using a tunnel between the Mobile Access Gateway (MAG) and the Local Mobility Anchor (LMA) [2], or (ii) direct routing: using the multicast infrastructure for sending/receiving the traffic [3]. Out of these methods, two possibilities are taken into account for the multicast functionality deployed in the MAG: MLDv2 Proxy [4][5] or multicast router (MR) [6]. IETF considers the tunnel-based MLD Proxy configuration as a base solution [2] to support multicast listener functions in PMIPv6 without modifying the mobility and multicast protocol standards. The

The research leading to these results has received funding from the European Community Seventh Framework Programme (FP7-ICT-2009-5) under grant agreement n. 258053 (MEDIEVAL project). This work was also supported by French Research project SYMPA. base solution can be also applied for the multicast source [7]. In this paper, we mainly focus on the multicast listener mobility.

Since the mobile node (MN) in the network-based mobility management is not aware of the mobility process, it cannot make multicast-related decisions. When a multicast listener moves to a new MAG, it has to wait to express its interest in subscribing to the multicast channels until it receives an MLD query. Thus, it experiences a certain delay in receiving multicast content due to the extra time related to the multicast service activation and MLD Query/Report transmission. That means the strict requirements of delay-sensitive applications like HD live video may not be fulfilled. One solution to this issue is using the context transfer mechanisms for exchanging multicast-related information between MAGs [8][9], which allows the new MAG (nMAG) to receive the multicast traffic in advance. Another possible solution is tuning the behavior of the IGMP/MLD for routers [10]. By varying the Query Interval (QI) and Query Response Interval (QRI), routers can tune the service activation time and join latency.

In this article, we present a testbed that allows providing the near-to-real experiment of multicast source and listener mobility in a PMIPv6 domain: a virtual PMIPv6 domain using a combination of User-mode Linux (UML) [11] and Network Simulator NS-3 [12]. The PMIPv6 entities (LMA and MAG) are UML virtual machines while the MNs are NS-3 nodes. Multicast mobility supporting for both the source and the listener can be enabled within this domain by using the deployment scenario as described in the base solution. Through this testbed, the experiment results show how to take advantage of the multicast context transfer mechanism to minimize the service disruption time. It also indicates that we can achieve a similar result by reducing the QRI. This reduction causes an increase in multicast-related signaling, which could influence the wireless link condition between MAG and MN. Especially, the problem will be more serious with a large number of multicast listeners. Based on the evaluation, the maximum number of listeners supported by one MAG is suggested depending on wireless link capacity.

The rest of this paper is organized as follows. Section II summarizes the problems and solutions proposed to support multicast mobility in PMIPv6. In Section III, the testbed implementation and the simulation scenarios are introduced. Section IV provides some analysis of multicast service 
disruption time, the simulation results, and the evaluation. Section V discusses the impact of MLD traffic on the wireless link and proposes the Bulk Context Transfer mechanism as an enhanced solution for group multicast mobility. Eventually, Section VI concludes this paper and provides perspectives for the future work.

\section{RELATED WORK}

\section{A. Multicast Support in PMIPv6}

As described in the previous section, two possible approaches of multicast mobility support in PMIPv6 are considered: (i) tunnel-based: using a tunnel between the MAG and the LMA, or (ii) direct routing: using the multicast infrastructure for delivering multicast traffic.

In the first approach [2][7][13], the multicast traffic is always routed via the mobile node's LMA. MLD proxy functions need to be deployed at MAGs with the upstream interface being configured to the corresponding mobile node's LMA which plays the role of an MR or an additional MLD proxy. As an MLD proxy, multicast data arriving from an upstream interface of an MLD proxy will be forwarded to the downstream interfaces which have appropriate forwarding states for this group. Thus, all multicast traffic passes through the MAG-LMA tunnel, just like unicast traffic. However, the presence of the tunnel raises some issues: (i) packet overhead, (ii) processing overhead, and (iii) non-optimal route [13].

The second approach takes advantage of the native multicast infrastructure for delivering multicast traffic, hence avoiding the tunneling overhead [3][7][13]. Moreover, the complexity of LMA is reduced since it does not have to deal with multicast traffic processing. Yet, this approach may require the multicast tree reconstruction during handover which results in significant service disruption. Some mechanisms are required to make sure that the multicast sessions continue right after the listener attached to nMAG and minimize the overhead in reconstructing the multicast trees [14].

\section{B. Multicast listener mobility and Service continuity}

Several strategies have been proposed to optimize the multicast service continuity when a listener is moving between MAGs. The first strategy [15] is based on the idea that the multicast subscription of the listener is only critical during handover, neither after nor before. The multicast membership of the active listener will be stored in the LMA (if necessary), and then the new MAG (nMAG) will interrogate the LMA to obtain the existing multicast subscription of the listener.

In the second strategy [9], the context transfer [8] is used to exchange the listener's active multicast subscriptions between the previous MAG (pMAG) and the new one. Two possible handover modes are considered: predictive and reactive mode. The difference between two modes is how nMAG gains knowledge of the listener's active multicast subscriptions. In the predictive handover, the pMAG will learn about the upcoming movement of the listener. After gaining knowledge of the on-going multicast subscriptions by using the explicit tracking function [10] or a general MLD Query, the pMAG sends it to the nMAG. In the reactive handover, the nMAG gets

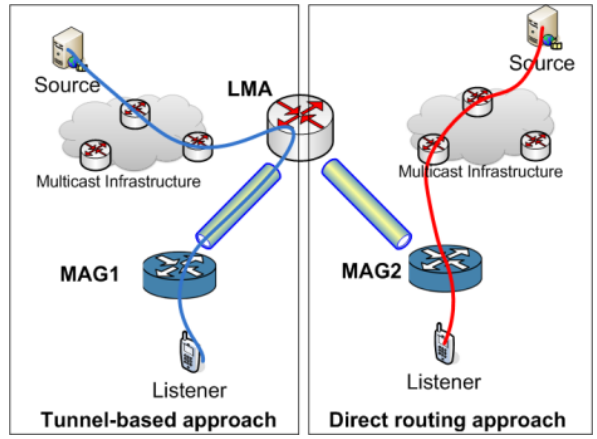

Figure 1. Multicast support in PMIPv6.

the listener's multicast subscriptions by using the regular MLD process. Then, the nMAG will join the necessary multicast groups on behalf of the listener.

The third strategy [10] is tuning the behavior of the IGMP/MLD for routers. In this paper, only MLDv2 is considered since we focus on the IPv6 network. By varying the QI and QRI, the routers can tune the service activation time and join latency. Slow multicast service activation following a join may incur an additional delay in receiving multicast packets in the nMAG. By reducing the QI and QRI, the service disruption time can be lower but result in the increase of the multicast-related signaling. In addition, the departure of the mobile host without leaving the group in the pMAG may cause the network resources waste.

Since PMIPv6 needs to be extended in the first strategy, up to now, only two strategies are considered from which two simulation scenarios will be made accordingly (see Section III).

\section{TeStBed IMPlEMENTATION AND SCENARIOS DESCRIPTION}

There are several methods to experiment the multicast mobility in PMIPv6 such as using real testbed or using simulation tools. First, to use a real testbed, we need at least three real machines: one plays the role of LMA while the others provide the MAG functionality. The challenge of this method is how to experiment the mobility of the sources and listeners, particularly when a mobility pattern is considered. For the second method, simulation tools such as Network Simulator NS-3, MATLAB, OMNeT++ can be used. However, this method, in some cases, fails to reflect the real experiment. In addition, some required components are still missing in these simulations e.g. PMIPv6, MLD proxy, and multicast router functions.

Due to the limitations of the above-mentioned methods, we provide an ultimate method using a virtualization based testbed - a combination of UML [11] and Network simulator NS-3 [12] that allows keeping the results close to the real experiment with insignificant efforts.

In our testbed, the PMIP entities are UML virtual machines while the Access Points (APs) and the MNs are NS-3 nodes. Using UML, many virtual machines can be run independently inside a real machine. Network Simulator NS-3 is used to emulate the wireless environment and the mobility of multicast nodes. Via the Tap Bridge model, the machine inside NS-3 can be made similar to a real machine. Also, the mobility patterns 


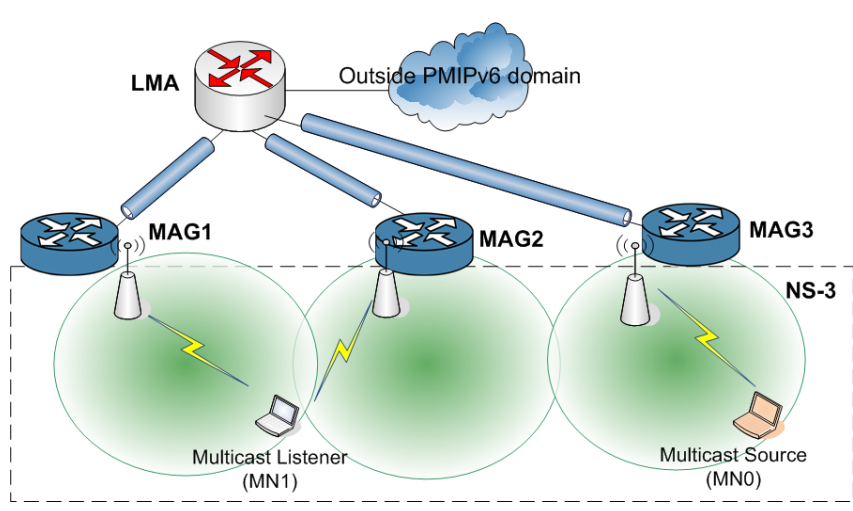

Figure 2. Virtual PMIPv6 Testbed.

can be used to make more flexible mobility of multicast nodes under NS-3.

\section{A. Description of the Testbed}

To clarify the influence of the utilization of multicast context transfer and tuning the behavior of the MLD for routers on multicast service disruption time, the other factors affecting the service disruption time should be fixed. Hence, thanks to the stability of multicast delivery tree, only the tunnel-based approach is considered. A reference topology for multicast support in a PMIPv6 domain is illustrated in Fig. 2.

The open source PMIPv6 implementation, named OAI PMIP [16], is used. The Linux kernel 2.6.38 which is recomplied to enable some required features for OAI PMIPv6 implementation, serves as the kernel for the PMIP entities. In OAI PMIPv6 implementation, the attachment/detachment phase for the MNs relies on SYSLOG message exchanged between Client (Access Point) and Server SYSLOG (MAG). Thus, the Client SYSLOG function is implemented in NS-3 and deployed at the APs.

As described in Section II, to enable multicast support in a PMIPv6 domain, MLD functions need to be deployed at the MAG while the multicast router functions are provided at the LMA. There are several implementations of MLD proxy such as McProxy [17], ECMH [18], etc. Though the former is newer, it only supports MLDv1. That is why ECMH is selected. Yet, some functions need to be added into ECMH to support the multicast source mobility. About the multicast router functions, the considered multicast routing protocols are PIM-SM [6] and PIM-SSM [19]. There are two potential

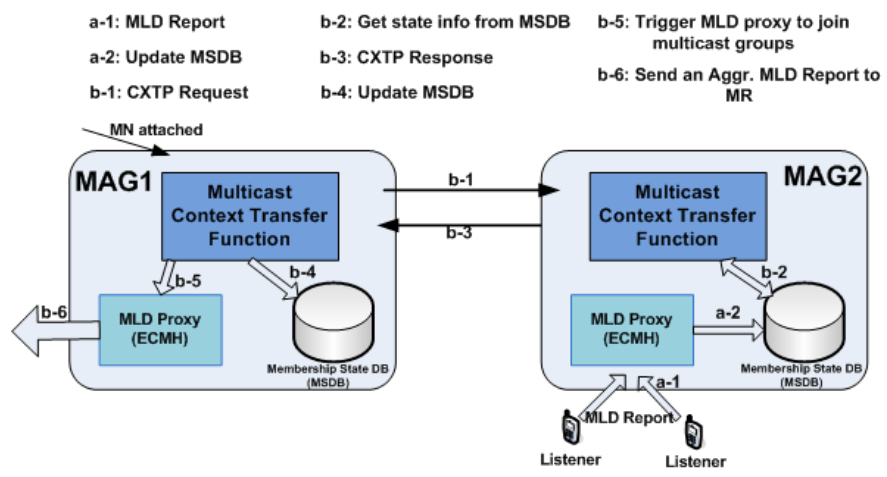

Figure 3. Implementation of context transfer and explicit tracking function. candidates providing PIM router functions: MRD6 [20] and XORP [21]. The first candidate is chosen because of its simplicity of deployment and configuration under UML.

We implemented MLDv2 protocol for Multicast Address Listeners [5] under NS-3 to enable the multicast capability of NS-3 nodes. Also, the multicast context transfer and explicit tracking function, as described in [22], are provided to facilitate the multicast listener mobility (see Fig. 3).

\section{B. Simulation Scenarios}

The simulations are executed by using a testbed which consists of one LMA, 3 MAGs and 2 MNs. The multicast source (MN0) is attached to MAG3 while the listener (MN1) is associated to MAG1. Acting as a multicast listener, MN1 is subscribed to the multicast session being broadcasted by the source. Focusing on the service disruption time during handover, two simulation scenarios are defined as follows:

- Scenario 1: tuning the behavior of the MLD for routers. In this scenario, the regular behavior of MLD protocol takes place while the QRI is varied to measure the service disruption time. Upon receiving an MLD Query at the new link, the MN1 replies by a regular MLD Report. Then, the nMAG sends an aggregated MLD Report to join the multicast group on behalf of the MN1. Thus, the multicast traffic originated by source is routed from LMA to listener via the new tunnel LMAnMAG.

- Scenario 2: using the multicast context transfer. When the multicast context transfer is used, by detecting the presence of a new listener, the multicast context transfer between nMAG and pMAG is executed, allowing nMAG to get the listener's active multicast subscriptions. As an MLD proxy, nMAG joins the multicast group on behalf of the listener, and forwards the multicast traffic to the listener.

To make sure that the simulation results reflect exactly the impact of the two strategies, the parameter QRI will be varied in both scenarios. According to [5][10], possible values of QRI using in the simulations are 10,5 and 2 seconds.

By now, to simplify the simulation, a simple mobility model is used: the listener moves between two MAGs with a fixed speed and a fixed direction. However, in the future, the mobility pattern will be applied to provide more flexible mobility of multicast listener. Also, the scenario in which many listeners are moving at the same time will be considered. To better evaluate the service disruption time, the simulation result is collected by repeating the simulation 30 times for each scenario.

\section{PERFormance ANALYSIS}

\section{A. Service Disruption Time Analysis}

The service disruption time for multicast service is defined as a period when a multicast listener cannot receive the multicast packets. Thus, as can be seen in Fig. 4 (a), the service disruption time can be split into three main contributions: 


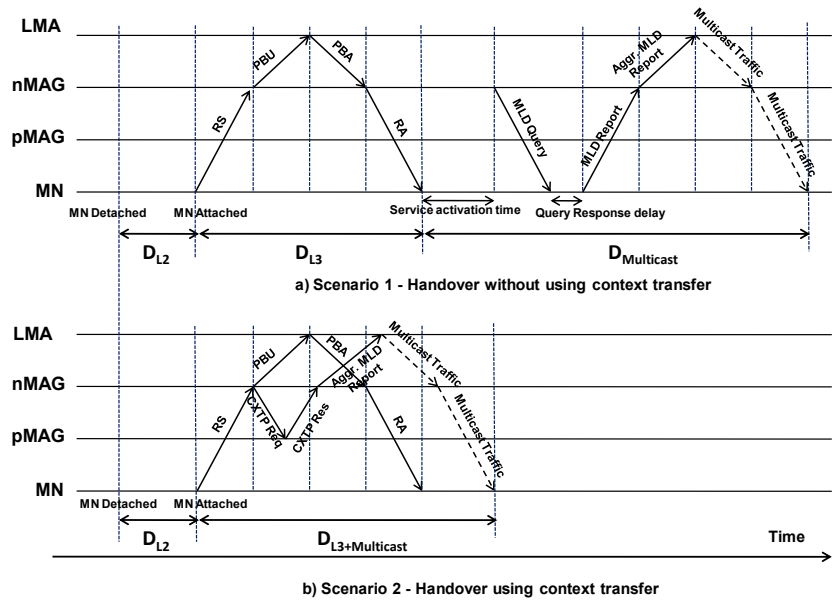

Figure 4. Signaling process for handover concerning multicast service.

- Layer 2 (L2) handover latency in WLAN $\left(\mathrm{D}_{\mathrm{L} 2}\right)$ which is due to the reattachment process from previous AP to the new one (including channel scanning, authentication, and association/re-association).

- Layer 3 (L3) handover duration $\left(\mathrm{D}_{\mathrm{L} 3}\right)$ caused by IPrelated procedures. In PMIPv6, it includes the time for mobility management procedures (movement detection and location update procedures).

- The delay due to the multicast-related procedures,

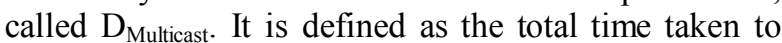
complete all the multicast-related procedures including the multicast knowledge gain, multicast subscription and transmission time for the first multicast packet from the multicast router to the $\mathrm{MN}$ after handover.

Let $\mathrm{t}_{\mathrm{X}, \mathrm{Y}}$ denote the delay between node $\mathrm{X}$ and node $\mathrm{Y}, \mathrm{t}_{\mathrm{MSA}}$ the multicast service activation time, and $t_{\mathrm{QRD}}$ the query response delay. For simplicity, we assume that the processing cost inside the LMA, MAG and MN is small enough to be ignored in the performance evaluation, and the delays are symmetric, i.e., $t_{M N, M A G}=t_{M A G, M N}$. Then the service disruption time is given by (see Fig. 4 (a)):

$$
\mathrm{D}_{\mathrm{Total}}=\mathrm{D}_{\mathrm{L} 2}+\mathrm{D}_{\mathrm{L} 3}+\mathrm{D}_{\text {Multicast }}
$$

Where $D_{L 3}$ and $D_{\text {multicast }}$ are given by:

$$
\begin{gathered}
\mathrm{D}_{\mathrm{L} 3}=2 \mathrm{t}_{\mathrm{MN}, \mathrm{nMAG}}+2 \mathrm{t}_{\mathrm{nMAG}, \mathrm{LMA}}, \\
\mathrm{D}_{\text {Multicast }}=\mathrm{t}_{\mathrm{MSA}}+\mathrm{t}_{\mathrm{QRD}}+3 \mathrm{t}_{\mathrm{MN}, \mathrm{nMAG}}+2 \mathrm{t}_{\mathrm{nMAG}, \mathrm{LMA}} .
\end{gathered}
$$

We suppose that MLD Queries are followed immediately the link-up event or the auto-configuration of IPv6 link-local address of a MN. Thus, the multicast service activation time can be ignored $\left(t_{\mathrm{MSA}}=0\right)$ [2]. As a result, in the simulations, the QI should be fixed while the QRI could be varied. As such, the total disruption time is:

$$
\mathrm{D}_{\text {Total_without_CXT }}=\mathrm{D}_{\mathrm{L} 2}+\mathrm{t}_{\mathrm{QRD}}+5 \mathrm{t}_{\mathrm{MN}, \mathrm{nMAG}}+4 \mathrm{t}_{\mathrm{nMAG}, \mathrm{LMA}} \text {. }
$$

Using the multicast context transfer and explicit tracking function, the multicast-related and L3 handoff procedures are operated in parallel as described in Fig. 4 (b). In this case, the service disruption time is calculated as follows:

$$
\mathrm{D}_{\text {Total_CXT }}=\mathrm{D}_{\mathrm{L} 2}+2 \mathrm{t}_{\mathrm{MN}, \mathrm{nMAG}}+2 \mathrm{t}_{\mathrm{nMAG}, \mathrm{pMAG}}+2 \mathrm{t}_{\mathrm{nMAG}, \mathrm{LMA}} .
$$

\section{B. Results and Evaluation}

\section{1) Comparison of two scenarios}

Fig. 5 describes the simulation results for service disruption time in terms of mean $(<x>)$ and standard deviation $\left(\sigma_{x}\right)$ for both scenarios. We can see that the service disruption time in the scenario 1 is absolutely higher than that in the scenario 2 due to the value of $t_{\mathrm{QRD}}$. As expected, the service disruption time in the former case decreases proportionally with the QRI, while almost keeping as a constant as the decreasing of QRI in the latter case.

The average value of service disruption time in the scenario 1 is $1378.0 \mathrm{~ms}(\sigma=643.4 \mathrm{~ms})$ in the best case (when QRI is set to $2 \mathrm{~s}$ ), which makes the impact of handover noticeable. If the multicast context transfer is used, in average, the service disruption time is around $366 \mathrm{~ms}$. Consequently, the handover impact on the quality of multicast stream is almost imperceptible.

The variation of service disruption time in both scenarios is clearly seen since it depends on several factors like scanning, association, authentication and $\mathrm{t}_{\mathrm{QRD}}$ (scenario 1). Even $\mathrm{t}_{\mathrm{QRD}}$ can spread out over the large interval $[0, \mathrm{QRI}],<\mathrm{t}_{\mathrm{QRD}}>$ is definitely higher than that of other delay types (L2 and L3). Hence, $t_{\mathrm{QRD}}$ is the crucial factor in the service disruption time.

\section{2) Service disruption time: Theoretical vs. Simulation} Results

In our analysis, $t_{\mathrm{MAG}}$, LMA, $\mathrm{t}_{\mathrm{pMAG}}, \mathrm{nMAG}$ and $\mathrm{t}_{\mathrm{MN}}$, MAG are assumed to be $10 \mathrm{~ms}, 10 \mathrm{~ms}, 12 \mathrm{~ms}$, respectively, according to the literature [23]. The value of $\mathrm{D}_{\mathrm{L} 2}$ varies over a range [0.1, 396] ms [24].

Fig. 6 describes the theoretical and simulation results. It is observed that the simulation results are in line with the theoretical analysis.

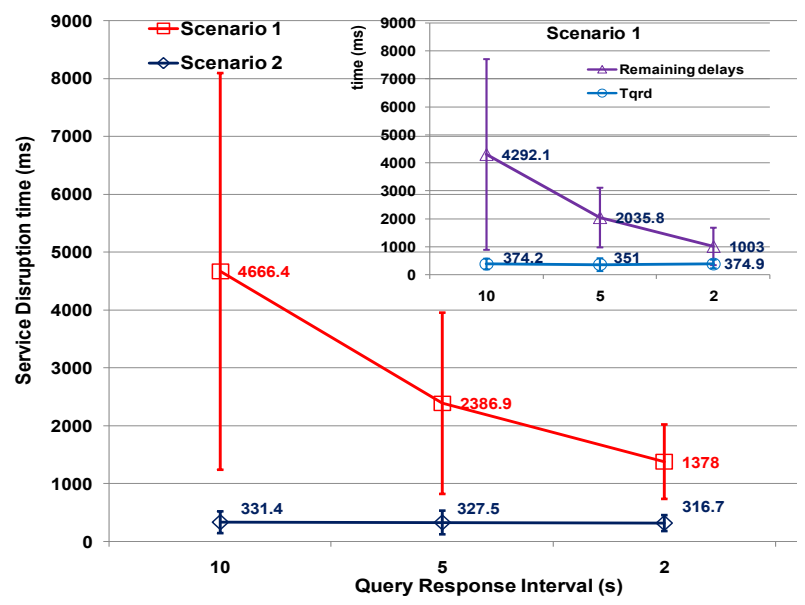

Figure 5. Service disruption time in two simulation scenarios. 


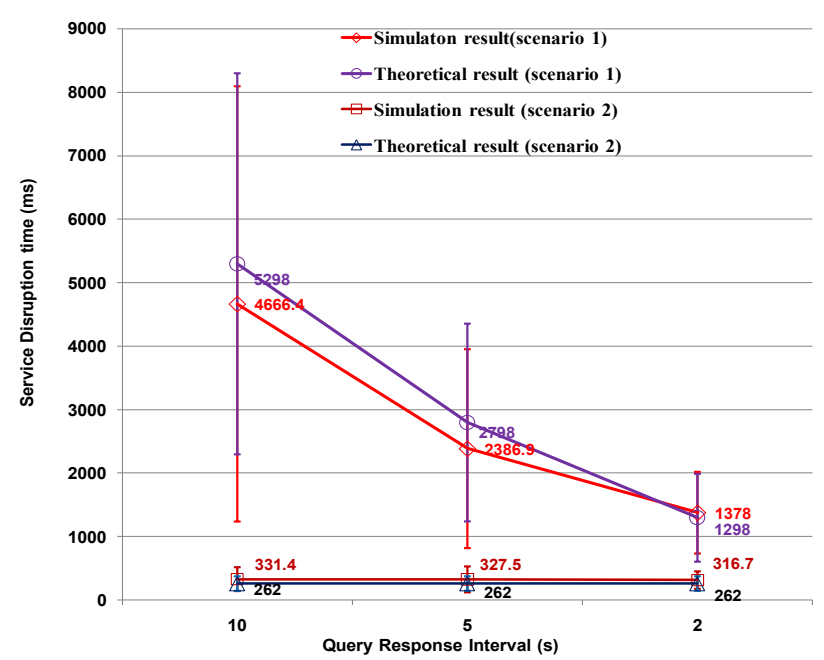

Figure 6. Service disruption time, theoretical and simulation results.

\section{DISCUSSION}

\section{A. Impact of QRI reduction on the wireless link condition}

If no multicast context transfer is used, the service disruption during handover can be clearly seen, even in the best case. To minimize the handover effect, we need to reduce the value of the query response delay $\left(\mathrm{t}_{\mathrm{QRD}}\right)$ by decreasing the $\mathrm{QRI}$. This reduction facilitates to achieve a seamless handover but makes the traffic more bursty. In this section, we measure the impact of QRI reduction on the wireless link. Based on that, the maximum number of listeners supported by one MAG is suggested, depending on the wireless link capacity.

Let $\mathrm{C}$ denote the uplink bandwidth capacity of the wireless link between $\mathrm{MN}$ and MAG (Mbps), $\mathrm{N}$ the number of multicast nodes attached to MAG and S the average size of MLD Report messages.

After receiving a MLD query (periodical query or a query caused by a link-up event), the multicast listeners reply by an MLD Report at the intervals chosen randomly from the range $[0, \mathrm{QRI}]$. During the period [0, QRI], there are N MLD Report messages generated on the link. Comparing with the scenario using context transfer, the number of signaling messages is dramatically increased (with context transfer, only 2 messages are exchanged between MAGs via a wired link). Thus, the required bandwidth for sending the MLD Report messages is:

$$
\mathrm{C}_{\text {required }}=\mathrm{N} \times \mathrm{S} / \mathrm{QRI} \text {. }
$$

Let $\sigma$ denote the effect of MLD traffic on the wireless link. It is calculated as the ratio of required bandwidth for MLD messages to the capacity of the link.

$$
\rho=\mathrm{C}_{\text {required }} / \mathrm{C}=\mathrm{N} \times \mathrm{S} /(\mathrm{QRI} \times \mathrm{C})
$$

To avoid the significant impact of the MLD traffic on the wireless link, the operator would keep the value of $\sigma$ as small as possible, for example $\rho \leq \rho_{0}$. From (4), by fixing the parameter $\mathrm{C}$ and $\mathrm{S}$, the maximum number of the multicast listeners attached on one MAG depends on QRI and $\rho_{0}$. It is given by:

$$
\mathrm{N}_{\max }=\rho_{0} \times \mathrm{QRI} \times \mathrm{C} / \mathrm{S} .
$$

From the theoretical results, $\mathrm{D}_{\text {Total CXT }}$, in the worse case, is $460 \mathrm{~ms}$. From (2), to achieve a similar delay without any context transfer, $t_{\mathrm{QRD}}$ must be less than or equal to a value of $359.9 \mathrm{~ms}$ (when $\mathrm{D}_{\mathrm{L} 2}=0.1 \mathrm{~ms}$ ). It is done by setting $\mathrm{QRI}$ to a value of $359.9 \mathrm{~ms}$. It was also proven by the simulation results that the mean and standard deviation of service disruption time are $305.34,180.34 \mathrm{~ms}$ respectively. With this parameter, Fig. 7 illustrates the impact of the MLD messages generated on the wireless link by varying the number of listeners attached to one MAG. It is noted that a typical PMIP deployment approximately allows for $5000 \mathrm{MNs}$ attached to one MAG [2].

For the same set of parameters, the maximum number of listeners supported by one MAG is depicted in Fig. 8 as a function of the wireless link capacity for some values of $\rho_{0}$ (see (5)). From this figure, we can determine the maximum number of listeners attached to one MAG in some cases, for example 802.11a, LTE, 802.11n as 130, 120, and 719 listeners respectively $\left(\rho_{0}=0.01\right)$ in order to minimize the service disruption time.

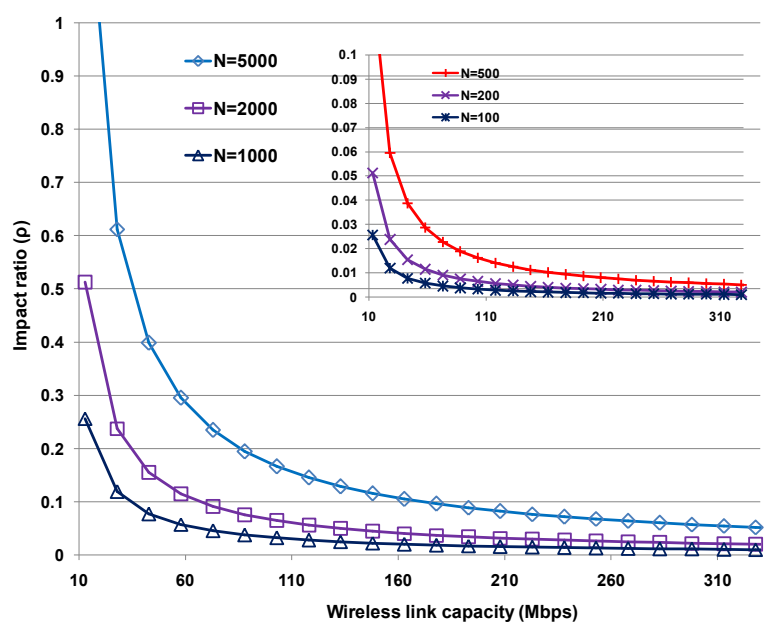

Figure 7. Impact of MLD messages generated on the wireless link.

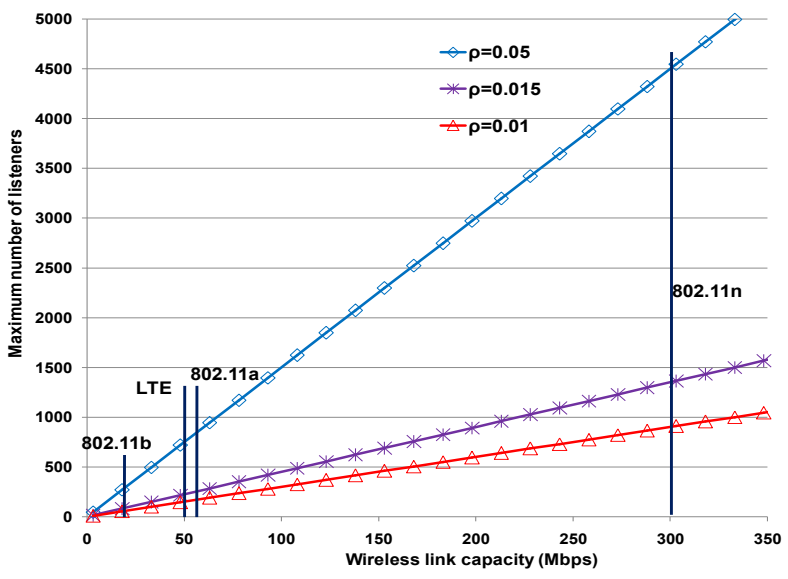

Figure 8. Maximum number of listeners vs. bandwidth capacity. 


\section{B. Bulk Context Transfer}

In the mobile environments, the utilization of the multicast context transfer would bring about a signaling overhead which is proportional to the amount of listener's handoff. To reduce the number of context transfer messages, we would like to propose an enhanced solution of context transfer for group mobility, called Bulk Context Transfer. This proposal can be used in a PMIPv6 domain applying Bulk Binding Update as described in [25].

We assume that there are $\mathrm{n}$ listeners belonging to the same group mobility $\mathrm{G}$ and moving between MAGs. When the first listener (MN1) is attached to the $\mathrm{nMAG}$, the $\mathrm{nMAG}$ will send a context transfer request message towards pMAG to get the MN1's active multicast subscriptions. Since the pMAG knows that the MN1 belongs to $\mathrm{G}$, it will embed all the multicast subscriptions related to this group in a context transfer response message and send it to nMAG. Upon receiving the multicast information, the nMAG will join the necessary multicast groups. Thus, the following listeners can receive the multicast traffic as soon as they configure their address at the new link. In case of using context transfer, the number of context transfer messages is $2 \mathrm{n}$, whilst this proposed solution needs only 2 messages. Moreover, the following listeners can get benefit from the available multicast traffic at the new link to reduce the service disruption time.

More precise evaluations will be provided in the next simulations when the spatial dependency mobility model such as Reference Point Group Mobility model is applied in NS-3.

\section{CONCLUSION AND FUTURE WORK}

This paper focuses on the effect of using the multicast context transfer and tuning the behavior of the IGMP/MLD for routers on handover performance of multicast listener mobility. The theoretical and simulation results show that through the utilization of multicast context transfer, the service disruption time can be reduced significantly without increasing the multicast-related signaling. We also observe that by tuning the behavior of the IGMP/MLD for routers, we can achieve a similar result, but make a noticeable multicast-related signaling increase. Thus, the impact of multicast-related signaling on the wireless link by the number of listeners is studied to suggest the maximum number of listeners supported by one MAG.

This paper also introduces a testbed that allows providing the near-to-real experiment of multicast source and listener mobility in a PMIPv6 domain. We also propose an enhanced solution for group mobility to reduce the number of context transfer messages exchanging between the MAGs.

Future research will aim at the performance evaluation of many approaches: tunnel-based versus direct routing, predictive versus reactive handover, multicast source mobility and the impact of the multicast context transfer when mobility pattern is applied.

\section{REFERENCES}

[1] S. Gundavelli, K. Leung, V. Devarapalli, K. Chowdhury, and B. Patil, "Proxy Mobile IPv6", RFC 5213, August 2008.

[2] T. Schmidt, M. Waehlisch, and S. Krishnan, "Base Deployment for Multicast Listener Support in PMIPv6 Domains", RFC 6224, April2011.

[3] JC. Zuniga, LM. Contreras, CJ. Bernardos, S. Jeon, and Y. Kim, "Multicast Mobility Routing Optimizations for Proxy Mobile IPv6", IETF Draft (work in progress), October 2012.

[4] B. Fenner, H. He, B. Haberman, and H. Sandick, "Internet Group Management Protocol (IGMP) /Multicast Listener Discovery (MLD)Based Multicast Forwarding ("IGMP/MLD Proxying")", RFC 4605, August 2006.

[5] R. Vida and L. Costa, "Multicast Listener Discovery Version 2 (MLDv2) for IPv6", RFC 3810, June 2004.

[6] B. Fenner, M. Handley, H. Holbrook, and I. Kouvelas, "Protocol Independent Multicast - Sparse Mode (PIM-SM): Protocol Specification (Revised)", RFC 4601, August 2006.

[7] T. Schmidt, G. Gao, H. Zhang, and M. Waehlisch, "Mobile Multicast Sender Support in Proxy Mobile IPv6 (PMIPv6) Domains", IETF Draft (work in progress), October 2012.

[8] D. Von Hugo and H. Asaeda, "Context Transfer Protocol Extension for Multicast", IETF Draft (work in progress), August 2012.

[9] T. Schmidt, M. Waehlisch, R. Koodli, and G. Fairhurst, "Multicast Listener Extensions for MIPv6 and PMIPv6 Fast Handovers", IETF Draft (work in progress), December 2012.

[10] H. Asaeda, H. Liu, and Q. Wu, "Tuning the Behavior of the Internet Group Management Protocol (IGMP) and Multicast Listener Discovery (MLD) for Routers in Mobile and Wireless Networks", RFC 6636, May 2012.

[11] User-Mode Linux, http://www.user-mode-linux.sourceforge.net.

[12] Network Simulator NS3, http://www.nsnam.org.

[13] MEDIEVAL Project, Deliverable D4.2: "IP Multicast Mobility Solutions for Video Services", June 2011.

[14] I. Romdhani, M. Kellil, H. Lach, A. Bouabdallah, and H. Bettahar, "IP Mobile Multicast: Challenges and Solutions", IEEE Communications Surveys \& Tutorials, vol. 6, no. 1, pp. 18-41, 2004

[15] LM. Contreras, CJ. Bernardos, and I. Soto, "PMIPv6 multicast handover optimization by the Subscription Information Acquisition through the LMA (SIAL)", IETF Draft (work in progress), December 2012.

[16] OAI PMIPv6, http://www.openairinterface.org/openairinterface-proxymobile-ipv6-oai-pmipv6.

[17] MCProxy, http://mcproxy.realmv6.org/.

[18] ECMH - Easy Cast du Multi Hub, http://unfix.org/projects/ecmh/.

[19] H. Holbrook and B. Cain, "Source-Specific Multicast for IP", RFC 4607, August 2006.

[20] MRD6, http://fivebits.net/proj/mrd6.

[21] XORP, http://www.xorp.org.

[22] MEDIEVAL Project, Deliverable D4.3: "Final Specification for mobility components \& interfaces", June 2011.

[23] A. Diab, A. Mitschele-Thiel, and K. Getov, "Analysis of Proxy MIPv6 Performance compared to Fast MIPv6", Local Computer Networks, 33rd IEEE Conference on, pp.579-580, October 2008.

[24] A. Mishra, M. Shin, and W. Arbaugh, "An Empirical Analysis of the IEEE 802.11 MAC Layer Handoff Process", ACM SIGCOMM Computer Communication Review, vol. 33, no. 2, pp. 93-102, April 2003.

[25] F. Abinader, S. Gundavelli, K. Leung, S. Krishnan, and D. Premec, "Bulk Binding Update Support for PMIPv6", RFC 6602, May 2012. 